Review

\title{
Multiscale Modeling for Host-Guest Chemistry of Dendrimers in Solution
}

\author{
Seung Ha Kim and Monica H. Lamm* \\ Department of Chemical and Biological Engineering, Iowa State University, Ames, IA 50011, USA \\ * Author to whom correspondence should be addressed; E-Mail: mhlamm @ iastate.edu; \\ Tel.: 515-294-6533; Fax: 515-294-2689.
}

Received: 15 December 2011; in revised form: 31 January 2012 / Accepted: 6 February 2012 /

Published: 10 February 2012

\begin{abstract}
Dendrimers have been widely used as nanostructured carriers for guest species in a variety of applications in medicine, catalysis, and environmental remediation. Theory and simulation methods are an important complement to experimental approaches that are designed to develop a fundamental understanding about how dendrimers interact with guest molecules. This review focuses on computational studies aimed at providing a better understanding of the relevant physicochemical parameters at play in the binding and release mechanisms between polyamidoamine (PAMAM) dendrimers and guest species. We highlight recent contributions that model supramolecular dendrimer-guest complexes over the temporal and spatial scales spanned by simulation methods ranging from all-atom molecular dynamics to statistical field theory. The role of solvent effects on dendrimer-guest interactions and the importance of relating model parameters across multiple scales is discussed.
\end{abstract}

Keywords: multiscale modeling; dendrimer simulations; host-guest chemistry

\section{Introduction}

Dendrimers have received increasing attention over the past several decades as attractive candidate materials for a variety of applications such as therapeutic delivery systems [1-5], imaging agents [4,6,7], templates for catalytic metal nanoparticles [8-10], and extraction agents for the removal of organic pollutants and toxic metals from water and soil [11-14]. The breadth across applications is attributed 
to dendrimers being well-defined, monodisperse nanostructures with tunable critical nanoscale design parameters [15,16], such as size, shape, rigidity, surface functionality, and solvent affinity. For this reason, dendrimers have been studied as hosts for a number of guest species: drugs [1-4], gene [17-19], contrast agents [7,20,21], metal ions [9-14], polymers [22], and organic pollutants [23,24]. Astruc and coworkers have written an exceptionally comprehensive review on dendrimers functioning as hosts [25].

A number of experimental approaches have been conducted to elucidate the interaction of dendrimers with guest molecules. The binding strength between dendrimer-guest complexes has been determined by isothermal titration calorimetry(ITC) [26-29], high performance liquid chromatography (HPLC) [30] and fluorescence spectroscopy [31-33]. Detailed conformations of dendrimers interacting with guest molecules have been studied by nuclear magnetic resonance(NMR) [34-36] and the size of the dendrimer-guest complexes has been measured by dynamic light scattering (DLS) [22,31]. For instance, supramolecular assemblies of polyamidoamine (PAMAM) dendrimers and phenanthrene, an organic pollutant, have been studied using fluorescence resonance energy transfer (FRET) to understand the interaction between dendrimers and guest molecules in aqueous solution [24]. Using FRET experiments, the most stable state for PAMAM-phenanthrene binding was observed at a $\mathrm{pH}$ of 8 and 1:2 molar ratio of phenanthrene to PAMAM. However, the fundamental binding mechanism between PAMAM dendrimers and phenanthrene molecules, such as preferred binding sites and why the binding was optimum for specific conditions, could not be explained by the experimental techniques alone. In another case, fullerenol-PAMAM assemblies have been studied using isothermal titration calorimetry, dynamic light scattering, and fluorescence spectroscopy to understand the binding mechanism [29]. These experiments measured the binding constant and determined the apparent stoichiometry between PAMAM dendrimers and fullerenols, but lacked a detailed molecular description of binding mechanism. Theoretical and computational approaches can complement fundamental experimental studies like these to facilitate rational materials design of dendrimer carriers.

Various theoretical and computational approaches have been used to examine molecular-scale phenomena that occur in dendrimer systems with guest molecules [37-39]. The use of a particular modeling methodology is driven by the characteristic time and length scales of the dendrimer-guest complex, and there can be multiple time and length scales that span orders of magnitude. For this reason, multiscale modeling approaches are often necessary to understand the phenomena of dendrimer-guest complexes since these methodologies are capable of describing macromolecular systems from length and time scales commensurate with electronic structure theory to length and time scales in the nanoscale regime [40].

Figure 1 shows multiscale modeling strategies for dendrimer-guest complexes in solution as a function of temporal and spatial length scales. From the bottom to the top of Figure 1, four computational approaches are described: (a) ab-initio; (b) fragment molecular orbital (FMO) method; (c) all-atom simulation; and (d) mesoscale simulation. At an intrinsic level, the nuclear coordinates and electronic structures in dendrimer-guest complexes are described described using ab-initio quantum mechanics and density functional theory (DFT) (Figure 1(a)). The configurational barriers of the dendrimer [41], the binding energies between dendrimers and guests, and the potential binding sites on the dendrimer, especially metal ions $[42,43]$, have been studied using these fundamental methodologies. However, this approach can be used for only the lowest generation dendrimers because it is computationally 
intractable to carry out these types of calculations for systems with more than 1,000 atoms. To reduce the computational cost at this time and length scale, several techniques are used: semi-empirical methods, and quantum mechanics/molecular mechanics. Recently, the fragment molecular orbital (FMO) method was introduced by Kitaura and coworkers to describe a large system at the $a b$-initio level (Figure 1(b)) [44,45]. There are many advantages for using FMO. Those that are particularly relevant for dendrimer-guest complexes are that pair interaction energy decomposition analysis can be used to see the partial interactions between fragments, and the polarizable continuum model [46] (PCM) can be used as an implicit solvation method with FMO [47]. Even though a complex of dendrimers with guest molecules can be studied at the quantum level using the fragment molecular orbital (FMO) method, there are limitations, such as difficulties with optimizing the structures at the same level of theory and achieving convergence of the energy. For this reason, the FMO approach is most effective when it is coupled with methods such as docking or classical molecular dynamics to model the interaction of dendrimers with guest molecules.

Figure 1. Illustration of the temporal and spatial scales in the complex system of polyamidoamine dendrimers (PAMAM) and phenanthrenes (Phe). There are four systems included in this figure: (a) PAMAM branch and Phe molecule (ab-initio); (b) fragments of G5 PAMAM dendrimer with 4 Phe molecules by a rainbow color notation (FMO); (c) G5 PAMAM dendrimer (All-atom simulation); (d) coarse-graining G5 PAMAM and 216 coarse-grained G5 PAMAM dendrimers with 216 Phe molecules (Mesoscale simulation).

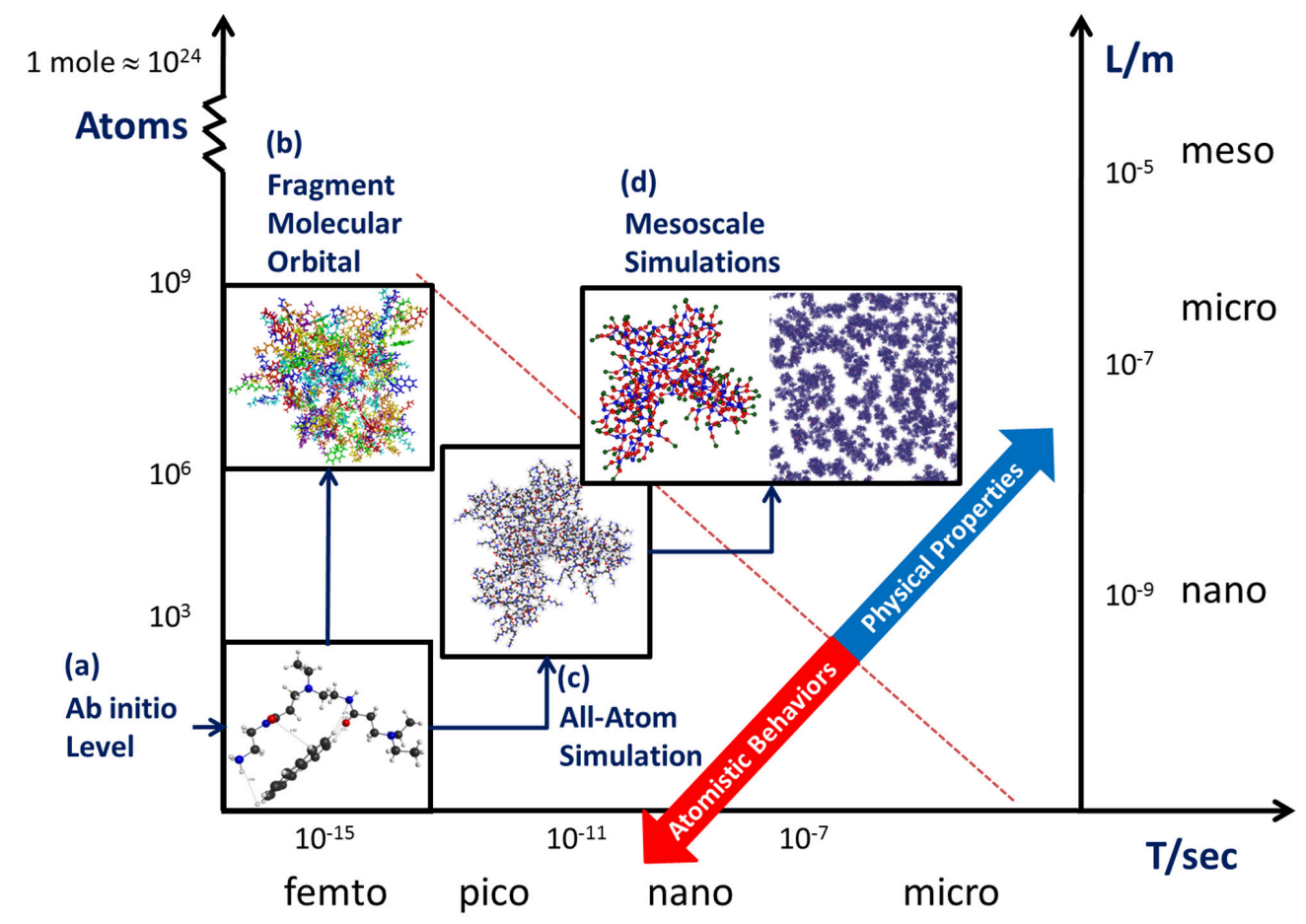

At the next higher scales of calculation, all-atom and mesoscale simulations have been widely applied to study the binding mechanism of dendrimers with guest molecules [24,38,39,48-51]. There are two main approaches for mesoscale simulations: coarse-grained "particle-based" simulations and statistical 
field theories. This review will cover computational methodologies that have been used to model dendrimers and dendrimer-guest complexes in solution using all-atom molecular dynamics (Section 2), coarse-grained molecular dynamics (Section 3) and statistical field theory (Section 4). Computational studies involving polyamidoamine (PAMAM) dendrimers are emphasized in this review because this class of dendrimer is the most widely investigated. Much of the outlook concerning multiscale modeling is not limited to a particular dendrimer chemistry, nor even to dendrimers themselves, but can be applied broadly to macromolecules in solution.

\section{All-Atom Molecular Dynamics Simulations}

All-atom molecular dynamics simulations are suited to modeling phenomena involving up to $10^{6}$ atoms over time scales on the order of tens of nanoseconds (Figure 1(c)). Thus, these simulations are routinely used to investigate conformational changes of a single dendrimer in explicit solvent with respect to dendrimer generation (size) and process conditions, such as temperature and solvent $\mathrm{pH}[52-55,62,63]$. All-atom simulations may also be used to investigate the dynamics of binding between of a single dendrimer and one or more ligands in explicit solvent [24,38,39,64].

\subsection{All-Atom Force Fields for Modeling Dendrimers}

Various force fields have been used to model dendrimers in solution with all-atom simulation. In the literature reviewed below, four force fields are commonly used: AMBER, CHARMM, CVFF, and Dreiding. The AMBER force field and generalized AMBER force field (GAFF) for organic molecules give good predictions for the binding between small organic ligands, nucleic acids, and proteins [56,57]. The CHARMM force field is specially developed for modeling the interactions between biological molecules such as proteins, nucleic acids, carbohydrates, and lipid membranes [58,59]. CVFF is a generalized valence force field optimized for organic molecules [60]. The Dreiding force field is a generalized force field for use with organic and biological systems [61]. As with all applications of molecular simulation, selecting an appropriate force field for modeling dendrimers and dendrimer-ligand complexes is critical to obtaining the correct fundamental behavior. In the case of PAMAM dendrimers, it has been frequently shown that predictions from molecular simulation for the swelling behavior of dendrimers as a function of solvent $\mathrm{pH}$ and salt concentration are very sensitive to the force field used in those simulations [52-54]. The hydrogen bonding parameters in the Dreiding III force field were recently optimized to prevent the swelling of PAMAM dendrimers at low $\mathrm{pH}$ and to predict radii of gyration that are consistent with small-angle neutron scattering experiments [63].

\subsection{The Physical Properties of Dendrimers in Solution}

Using all-atom molecular dynamics simulation, the effect of solvent $\mathrm{pH}$ on the PAMAM dendrimer was first considered by Baker and co-workers [65]. In this study, the primary and tertiary amines in the PAMAM dendrimers were protonated to model low, neutral, and high $\mathrm{pH}$ effects of PAMAM dendrimer based on $\mathrm{pH}$ titration data, and the CVFF force field was used. The solvent was modeled implicitly and this caused the estimations of the radii of gyration for the dendrimers to be higher than those obtained from experiments. 
Maiti et al. used atomistic molecular dynamics simulation to study the $\mathrm{pH}$ response of generation 4-6 PAMAM dendrimers [52] and a generation 8 PAMAM dendrimer [62] with explicit water molecules. From these calculations, the structure of the PAMAM dendrimer was shown to swell, with the branches extending as the solvent $\mathrm{pH}$ decreased. The open structures occur due to the electrostatic repulsions among the protonated tertiary (low $\mathrm{pH}$ ) and primary amines (low and neutral $\mathrm{pH}$ ) of the PAMAM dendrimers and due to the counterions and solvent molecules penetrating into the more open interior of the dendrimer at lower solvent $\mathrm{pH}$. This $\mathrm{pH}$ responsive phenomena of PAMAM dendrimers in solution makes them attractive candidates for applications requiring controlled encapsulation and release, such drug delivery and environmental remediation.

Maiti and coworkers also carried out 10-20 ns atomistic molecular dynamics to see the counterion distribution and calculated the $\zeta$ potential, which characterizes the degree of the electrical charge at the double layer, as a function of generation (3 to 7) for PAMAM dendrimers at neutral pH [48]. Such analysis is useful for understanding the electrostatic binding between PAMAM dendrimer and nucleic acids, which will be discussed further in the next subsection. Upon analyzing the counterion distribution, they found that the counterion density is higher in the interior regime of the dendrimer for higher generations. This can be explained by recognizing that the electrostatic repulsions inside of the dendrimer increase for higher generations because the amount of backfolding increases with increasing dendrimer generation $[2,52,66]$. To screen this electrostatic penalty, the counterions penetrate inside the dendrimer, hence, the concentration of counterions increases. They predicted the $\zeta$ potential of the dendrimer using the counterion density profile, and found that the $\zeta$ potential slowly increases with an increase of dendrimer generation, even though the surface potential exponentially increases. Thus, these simulations predict that the increased concentration of counterions does not affect the $\zeta$ potential of the dendrimer. The observation of $\zeta$ potential using molecular dynamics correspond to the results obtained for colloidal particles using Monte Carlo simulations and the Poisson-Boltzmann theory. Because the diffusion properties of carriers (hosts) are highly correlated to drug delivery performance, Maiti and coworkers studied the self-diffusion of dendrimers up to generation 8 for different solvent $\mathrm{pH}$ [67]. In this work, they found that PAMAM dendrimers did not follow the scaling laws of the Stokes-Einstein relation for diffusion. Because PAMAM dendrimers are flexible macromolecules with lots of interior cavities, water and ions penetrate to the interior of the dendrimers. Similar observations are made for the dynamics fractal aggregates in solution, where the ratio of hydrodynamic radius to radius of gyration as a function of aggregate mass varies with respect to the fractal dimension of the aggregate [68]. This has been attributed to the fact that the solvent accessible cavity of the solute molecule (in this, case dendrimer) varies based on the fractal dimension, and thus the diffusion coefficient also varies. Based on the different diffusion behavior observed for dendrimers compared to linear polymers, it appears necessary to treat water and counterions explicitly when modeling dendrimers in solution.

Recently, Goddard and coworkers modified the Dreiding III force field to more accurately model the $\mathrm{pH}$ responsive structural changes observed in small-angle neutron scattering experiments on PAMAM dendrimers. In the experiments, the observed radius of the gyration $\left(R_{g}\right)$ for a generation 4 PAMAM dendrimer was found to be independent of solvent $\mathrm{pH}$ [63]. Using new hydrogen bonding parameters obtained from quantum mechanics calculations, the molecular dynamics simulations confirmed that there 
was no change in radius of gyration and predicted that the internal distribution of atoms in the dendrimer varied with solvent $\mathrm{pH}$, from a "dense core" (high $\mathrm{pH}$ ) to a "dense shell" (low $\mathrm{pH}$ ).

\subsection{The Physical Properties of Complex Systems of Dendrimers with Guest Molecules in Solution}

All-atom molecular dynamics simulations have frequently been used to model the direct interactions of dendrimers with guest molecules or ligands. The types of guest molecules considered in this review will include low molecular weight organic molecules [24,29,50,51], and nucleic acids [38,69-73]. The review of interactions of dendrimers with lipid membrane has been clearly explained in Reference [37].

Dendrimer-drug complexes have generated a lot of interest due to the need for understanding the fundamental mechanisms for encapsulation and release of drug payloads inside a dendrimer carrier. Tanis and Karatasos [50] studied ibuprofen, a nonsteroidal anti-inflammatory drug, as a guest molecule bound to a G3 PAMAM dendrimer at different solvent $\mathrm{pH}$ conditions using atomistic molecular dynamics simulations with GAFF [56,57] for PAMAM and ibuprofen and TIP3P [74] for water. These simulations showed that hydrogen bonding between the hydrogen atom in the amide group on the dendrimer and the oxygen atom in the carbonyl group on ibuprofen was the primary interaction between the two species at neutral $\mathrm{pH}$. At high $\mathrm{pH}$, when ibuprofen is ionized due to the deprotonation of the carboxyl group, electrostatic interactions between between the ibuprofen and the primary amine groups was observed to be the primary interaction. No significant interaction between dendrimers and ibuprofen was observed in the simulations at low $\mathrm{pH}$. At this condition, electrostatic repulsions between the protonated tertiary and primary amines on the dendrimer leads to a more open structure that allows for penetration of water. Under this circumstance, the simulations showed that ibuprofen molecules formed clusters and diffused away from the dendrimer instead of hydrogen-bonding to the amine groups. Shi and coworkers studied 2-methoxyestradiol (2-ME), a potential anticancer agent, with modified PAMAM dendrimers at pH 5. The terminal group chemistries that were considered included amine, acetyl, hydroxyl, and carboxyl groups [51]. All-atom molecular dynamics using the CVFF force field for PAMAM and 2-ME and an implicit model for water were used to model the binding positions of 2-ME molecules in the dendrimer. The simulations showed that the structures of the dendrimers with amine, acetyl, and hydroxyl surface groups were open at low $\mathrm{pH}$, which presumably facilitates the release of 2-ME. Calculating the free energy of binding between the PAMAM variants and 2-ME would provide confirmation about the postulated release characteristics of these dendrimers. In contrast, the structures of the dendrimers with carboxyl surface groups were compact and encapsulated 2-ME at low $\mathrm{pH}$.

Similar behavior was reported by our group in a study of the phenanthrene-G5 PAMAM dendrimer complex using Drieding force field [24]. In this study, more phenanthrene molecules were bound to the G5 PAMAM dendrimer at neutral $\mathrm{pH}$ than at low $\mathrm{pH}$, even though the dendrimer structure is more open at low $\mathrm{pH}$ and thus there are more cavities in the interior of the dendrimer (Figure 2). The main reason of the high efficiency of the binding at neutral $\mathrm{pH}$ is that the hydrophobic interactions were the driving force for hydrophobic phenanthrene molecules to penetrate into the interior of the PAMAM dendrimer compared to a low $\mathrm{pH}$ case. Recently, our group has investigated complex formation between fullerenol, a fullerene derivative, and PAMAM dendrimers at neutral pH using GAFF [29]. The binding capacity of G1 and G4 PAMAM dendrimers for fullerenol was determined by molecular dynamics simulations. Using umbrella sampling simulations, the binding constant between PAMAM dendrimer and fullerenol was predicted 
to be independent of dendrimer size. However, the binding capacity of the G4 PAMAM dendrimer was 20-fold that of the G1 PAMAM dendrimer (Figure 3). The main reason is that the surface area of G4 PAMAM dendrimers is larger than that of G1 PAMAM dendrimers, thereby increasing the probability for binding fullerenol in G4 dendrimers compared to G1 dendrimers. After analyzing the types of hydrogen bond pairs formed during binding, the greatest fraction of hydrogen bond events occurred between the primary amines on the dendrimer and the oxygen on the hydroxyl group of fullerenols, which supports the increased surface area hypothesis.

Figure 2. Representative images of G5-PAMAM dendrimer with 25 of phenanthrene molecules after $1 \mathrm{~ns}$ as an atomistic molecular dynamics: (a) low $\mathrm{pH}$ (b) neutral $\mathrm{pH}$ (c) high $\mathrm{pH}$. The dark red arrows indicate the stacked Phe molecules. Reproduced from Ref. [24] with permission from the PCCP Owner Societies.

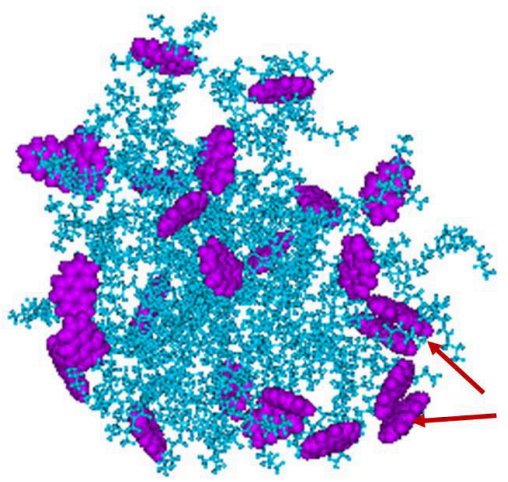

(a)

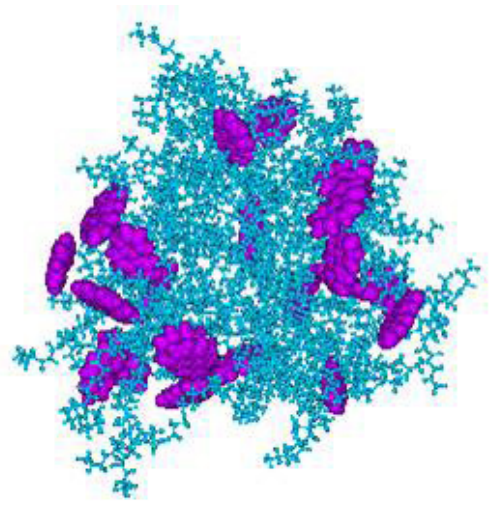

(b)

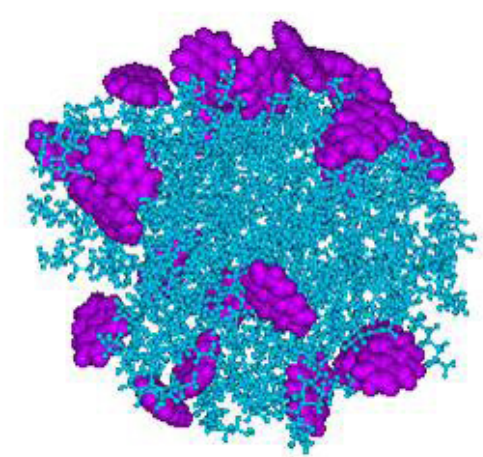

(c)

Figure 3. Interaction of PAMAM dendrimers with fullerenols at neutral $\mathrm{pH}$ : (a) Two fullerenols in proximity to one G1 dendrimer within $1.5 \mathrm{~nm}$ of the center of mass of the dendrimer (b) 21 fullerenols in proximity to one G4 PAMAM dendrimer within $3.5 \mathrm{~nm}$ of the center of mass of the dendrimer.

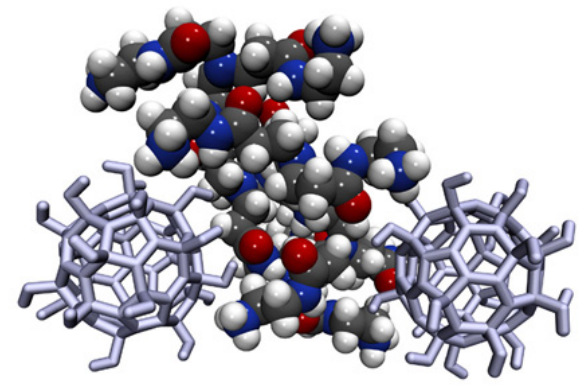

(a)

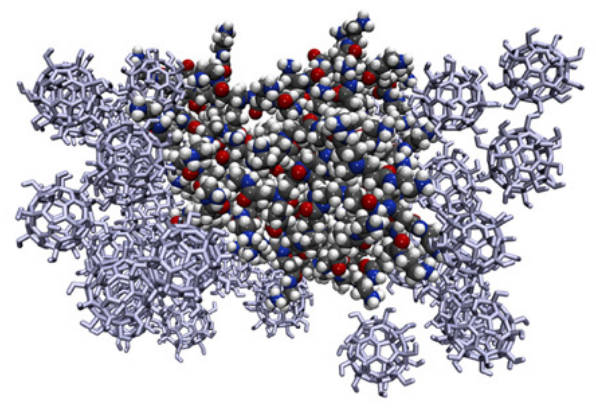

(b)

Dendrimers have been explored as a non-viral vector for gene therapy and that has motivated modeling studies of the binding mechanism between dendrimers and DNA [2]. Dendrimers and proteins have similar physical properties and hence, complexes of DNA and dendrimers have also been investigated 
as model systems for gaining insight about the fundamental binding interactions between proteins and genes. Maiti and Bagchi carried out all-atom molecular dynamics simulations using the Dreiding force field for PAMAM dendrimers and the AMBER95 force field for DNA to model the complex formed between a 38 base single-stranded DNA (ssDNA) and dendrimers of generations G2-G4 [69]. They included explicit water and counterions with the ssDNA-PAMAM complex, whereas the Debye-Hückel approximation had been applied to model the electrostatic interactions between DNA and dendrimers in the previous studies $[64,75]$. In this study, the binding interaction between PAMAM dendrimers and ssDNA was divided into three contributions: (i) the bending energy (entropic effect); (ii) the electrostatic energy; and (iii) the base pairing and stacking energy (enthalpic effect). For low generation (G2, G3) PAMAM dendrimers, the surface charges on the dendrimer were not enough to neutralize ssDNA, so the electrostatic interaction between dendrimers and ssDNA was not stronger than the bending interaction. Therefore the dendrimers did not cause ssDNA to adopt a coiled confirmation. However, in the higher generation (G4) PAMAM dendrimer, ssDNA was neutralized by the protonated amines of the dendrimer, so the enthalpic gain from the electrostatic energy overcame the entropic loss in the bending energy and ssDNA coiling was observed. They also calculated the free energy surface as a function of the distance between a dendrimer and ssDNA, and as a function of a parameter defined as the summation of the distance between each phosphate site and the center of mass of the dendrimer. Based on the free energy calculation, they explained the stability of the coiled ssDNA-dendrimer complex. Maiti and coworkers have also simulated complex formation between a 38 base pair double-stranded DNA (dsDNA) and generation (G3-G5) PAMAM dendrimers [38]. In this study, they proposed that the critical variable for wrapping nucleic acids around dendrimers is the charge ratio, which is defined as the number of positive amines in a dendrimer divided by the number of negative phosphates in a nucleic acid. The simulations showed that dsDNA completely wrapped around a G5 PAMAM dendrimer when the charge ratio is over 1 (1.64) even though the a 38 base pair dsDNA has a length short enough ( $13 \mathrm{~nm}$ ) considered to be a rigid rod (Figure 4). In the case of G3 and G4 PAMAM dendrimers (charge ratio $<1$ ), the dendrimer did not neutralize the dsDNA, so the dsDNA did not totally wrap the dendrimers like what was observed with the G5 PAMAM dendrimer. In addition, the lower generation (G3 and G4) dendrimers were deformed during the interaction with dsDNA, presumably due to the smaller surface area of the dendrimers. They also investigated the stability of the dsDNA-dendrimer complex using helicoidal parameters (rise, roll, twist, tilt, shift, slide) to characterize the overall backbone structure of the dsDNA in the complex and it was found that the G4 dendrimer-dsDNA complex was more stable than the G5 dendrimer-dsDNA complex. Free energies of the complex were calculated using the molecular mechanics Poisson Boltzmann surface area (MM-PBSA) method with two thermodynamic models for the entropy calculation. The free energies of the complex showed that the binding strength increases with higher generation of dendrimer and that the binding energy per protonated primary amine (dendrimer) was maximized for the G4 dendrimer, suggesting that the G4 dendrimer could be the optimum candidate for gene therapy applications. Mills et al. performed molecular dynamics simulations with the CHARMM 27 force field to study the binding between a dendrimer and dsDNA [70]. In this work, the local environment effects, such as deformation and the electrostatic interactions, were characterized and related to single molecule pulling experiments. The potential of mean force for a G3 PAMAM dendrimer-dsDNA ( $24 \mathrm{bp}$ ) complex was 
calculated by umbrella sampling and used to derive a mesoscopic stochastic model based on a Monte Carlo method. From the model they calculated the mesoscale force-extension curves.

Figure 4. (a) Structure of the DNA-G5 dendrimer complex during various stages of complex formation at the interval of a few nanoseconds (b) Time evolution of the radius of gyration $\left(R_{G}\right)$ of DNA, the dendrimer, and the complex for the complexation with G3, G4 and G5 dendrimers at neutral pH. Reprinted with permission from [38]. Copyright 2011 American Chemical Society.

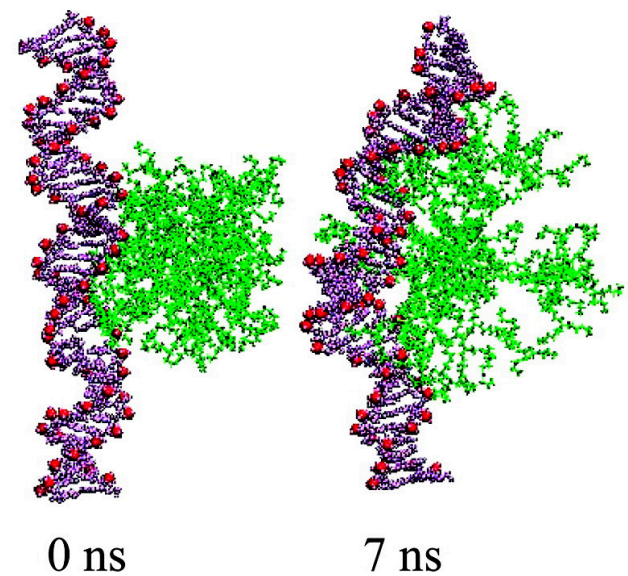

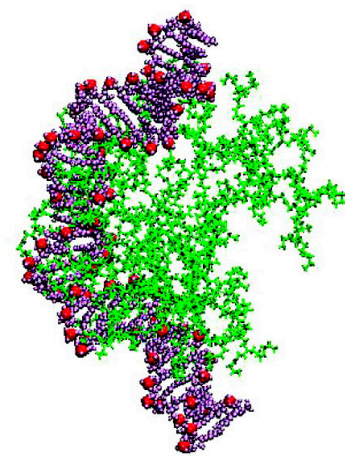

$18 \mathrm{~ns}$

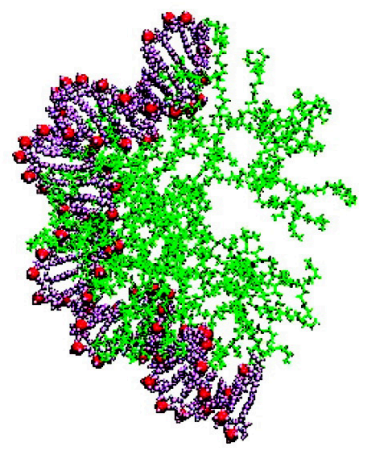

$25 \mathrm{~ns}$

(a)
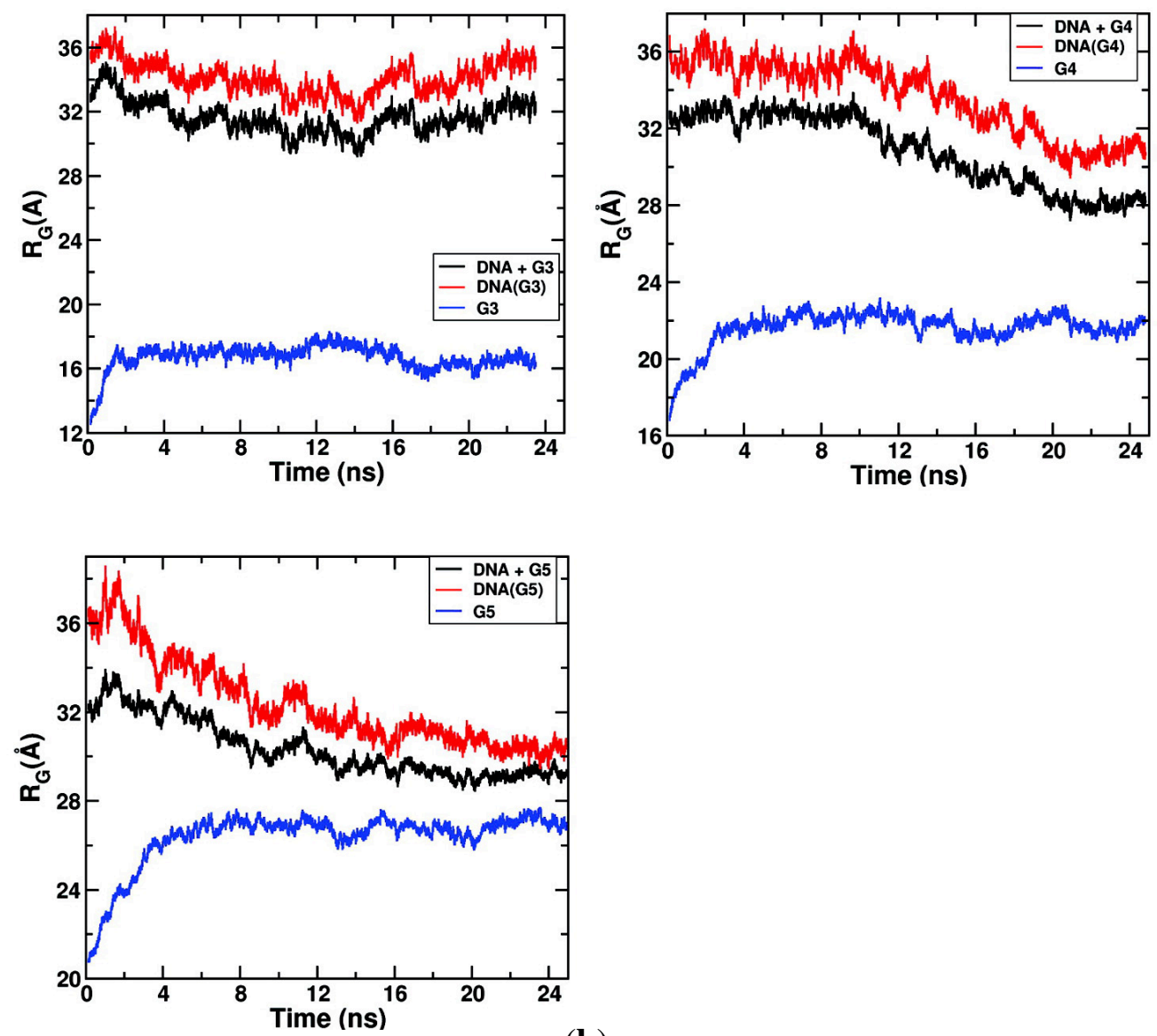

(b) 
Several all-atom molecular dynamics simulations studies have investigated dendrimer-RNA complexes. Pavan et al. investigated the G4-G6 PAMAM dendrimer-Firefly Luciferase (GL3) siRNA complex in solution [76]. The AMBER force field was used and the parameters for the PAMAM dendrimer atom types were derived using ab-initio calculations. The force field parameterization was validated against measurements of the hydrodynamic radius of PAMAM dendrimers using dynamic light scattering. The binding energies were calculated by the MM-PBSA method with normal mode analysis for the entropy. To investigate the flexibility of the binding behavior, an energetic flexibility (EF) index is defined as the ratio of the enthalpic contribution $(\Delta H)$ to the entropic contribution $(T \Delta S)$ of the binding energies. Since higher enthalpic and lower entropic contributions to the binding indicate strong attractions between RNA and a dendrimer, the authors saw a correlation between strong binding affinity and a higher EF index. Of particular note is that when binding data are interpreted within the scheme of the EF index, it was found that the binding efficiency for G4 PAMAM dendrimers does not decrease with decreasing solvent $\mathrm{pH}$ as one might expect, but the binding efficiency for the higher generation G6 PAMAM dendrimers does decrease as solvent $\mathrm{pH}$ is lowered. This suggests that the structure of G4 PAMAM is better able to accommodate the loss of entropic degrees of freedom (presumably through increased "flexibility") upon binding than its G6 PAMAM counterpart. Vasumathi and Maiti studied G3 and G4 PAMAM dendrimer-siRNA (21 bp) complexes using the Dreiding force field for PAMAM dendrimers and the AMBER03 force field for siRNA [72]. In this study, they considered the effects of counterion distribution, salt concentration, and the number of the dendrimer molecules involved in the complex. In these simulations, it was observed that a G4 PAMAM dendrimer gained more entropy from releasing $\mathrm{Na}^{+}$ions to bind with siRNA than the G3 PAMAM dendrimer. The simulations predicted that as salt concentration increases, the binding affinity decreases because the salt ions screen the favorable electrostatic interactions between PAMAM dendrimers and siRNA. In addition, they calculated the binding energy for the complex using the MM-PBSA method. As with the computational studies about dendrimer-dsDNA complexes discussed above, they found that the binding affinity is correlated to the charge ratio between the dendrimer and siRNA. To understand the stability of the binding, the radial distribution function was analyzed between the primary and tertiary amines in the dendrimer, sorted by the subgeneration of dendrimer and the phosphates in backbone of siRNA. From this analysis, it was found that the first peak of the curve is related to the protonated primary amines, and the second peak is related to the wrapping of siRNA and hence, the stability. Concerning on the basis of the radial distribution function analysis, the two G4 dendrimer-siRNA complexes were not stable even though the charge ratio indicates the binding affinity would be stronger than any other case considered.

All-atom simulations provide detailed information such as the binding locations, the hydrogen bonding pairs, and diffusion coefficients. However, if one wishes to consider multiple macromolecular hosts, the system is beyond the computational limit of all-atom simulations. In addition, to observe phenomena on longer characteristic time scales, up to $\mu$ s, the atomistic scale is not feasible.

\section{Coarse-Grained Simulations}

Coarse-grained molecular dynamics simulations are an effective strategy for obtaining large-scale structural information for dendrimer-guest assemblies, because the reduction in degrees of freedom permits computational investigations of length and time scales much larger than is possible with 
all-atom molecular models. The freely jointed chain model is one of the coarse-grained methodologies used to describe the behaviors of dendrimers in solution [77-80]. The advantage of this approach is that the key physical details governing the observed macroscopic properties can be easily extracted from the simulation predictions. However, the intermolecular interactions for this class of model are phenomenological and do not directly originate from the all-atom force fields or the thermodynamic properties of a specific dendrimer chemistry. To maintain chemical fidelity in coarse-grained molecular simulations, alternative multiscale coarse-graining strategies have been applied. In these multiscale strategies, most coarse-grained approaches can be classified into one of two categories. In the first category, termed indirect parameterization, are methods in which the potential parameters of a pre-selected analytical form are optimized by calibration against thermodynamic or structural properties. An example is the MARTINI force field for biological molecules [81,82], whose parameters are based on oil/water partitioning coefficients. In the second category, called direct parameterization, the coarse-grained potentials are determined from all-atom molecular dynamics simulations. One example is the force matching method [49,83-85].

In addition to coarse-grained molecular dynamics, Brownian dynamics and dissipative particle dynamics have been used to model dendrimers in solution with lower resolution molecular models [86-88]. The Brownian dynamics method has been widely used to study the interaction of a linear polyelectrolyte with oppositely charged dendrimers [86,87]. To describe the electrostatic interactions, the Debye-Hückel approximation has been applied in the Brownian dynamics method. Since explicit counterions should be considered in condensed systems to accurately model the counterion distribution, only dilute conditions may be modeled with this approximation. Given this limitation, this approach has been applied to study complexes of polyelectrolytes and dendrimers. Dissipative particle dynamics simulations, which are based on stochastic methods that permit a much longer time step, can be carried out for much longer time scales than atomistic or coarse-grained molecular dynamics simulations. Dissipative particle dynamics has been use for a charged dendrimer on a lipid membrane, including the effect of explicit counter ions, to model dynamics over $15 \mu$ s [88].

\subsection{The Physical Properties of Dendrimers in Solution}

Tian et al. studied the structure and the size of a charged dendrimer as a function of the counterion valency and different salt concentrations using a freely jointed bead-spring model for G4 cationic dendrimer [80]. In this study, they found that the strong electrostatic interaction from high valence ions neutralized the negatively charged surface group of the dendrimer and reduced the electrostatic repulsion compared to monovalent ions.Thus, the high valency counterions such as divalent and trivalenteffectively cause an osmotic pressure drop in the interior region of the dendrimer. In addition, the conformations of the dendrimer changed from extended to collapsed to a weak swollen state with increasing concentration of multivalent salt ions. These intriguing results warrant further investigation of dendrimer-polyelectrolyte complexes, by both experimentation and by all-atom molecular simulation.

Maiti et al. developed a generic coarse-grained model for PAMAM dendrimers to describe higher generations of dendrimers [89]. The mapping of coarse-grained sites to atoms is similar to the model developed by Lee and Larson [90] (Figure 5). Coarse-grained parameters for non-bonded and bonded potentials were derived using an all-atom molecular dynamics simulation of a G6 PAMAM 
dendrimer in the gas phase. The results obtained with this potential compare well with previous all-atom simulations [91] of PAMAM in the gas phase (poor solvent) but the potential is incapable of providing insight about behavior in water (good solvent).

Figure 5. (a) Mapping of dendrimer PAMAM segments into coarse-grained beads; (b) The same segment in PAMAM has different bond lengths and we apply special protocol to label each segment. The distance between the center of beads $\mathrm{O}$ and $\mathrm{N}$ is different from the distance between the center of beads $\mathrm{N}$ and $\mathrm{S}$, although beads $\mathrm{O}$ and $\mathrm{S}$ represent exactly the same segment (-CH2-CO-NH-CH2). Reprinted with permission from [89]. Copyright 2009 by the American Institute of Physics.

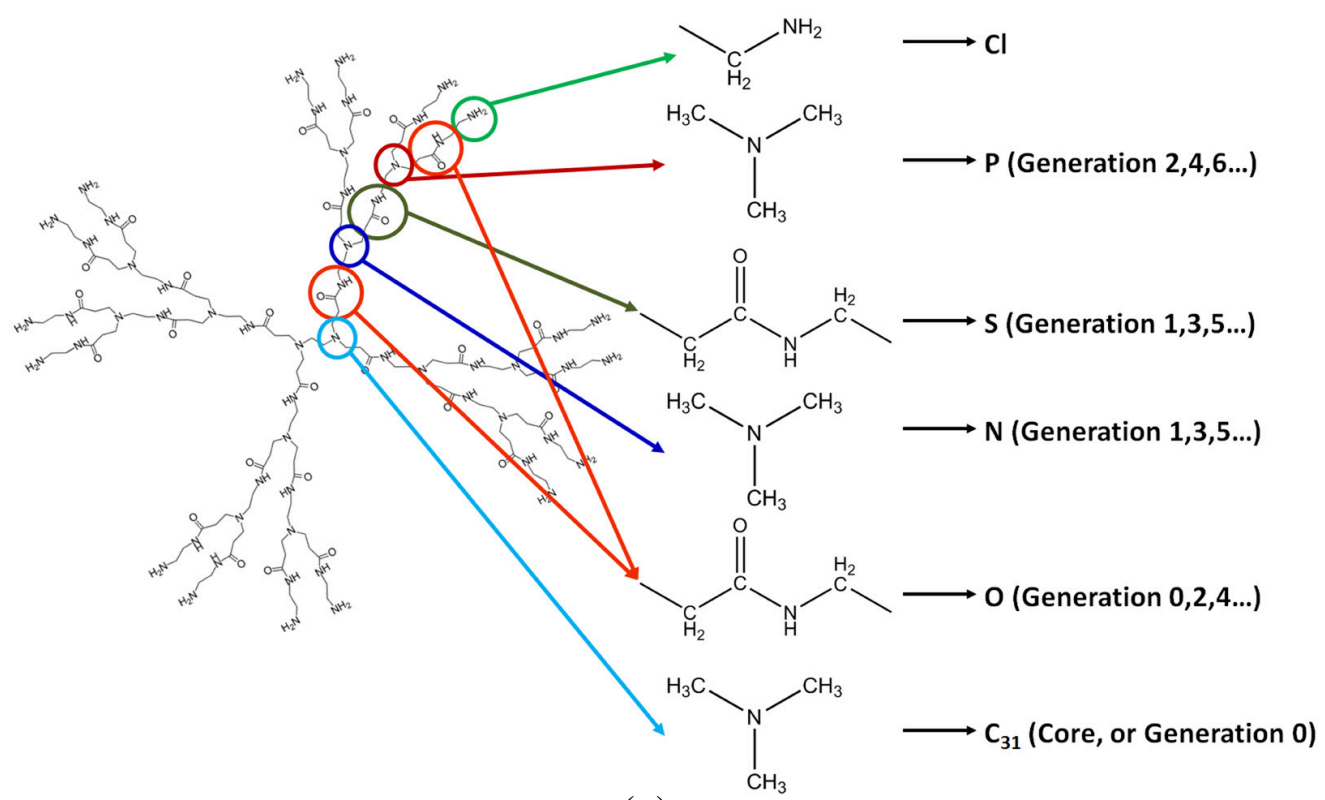

(a)

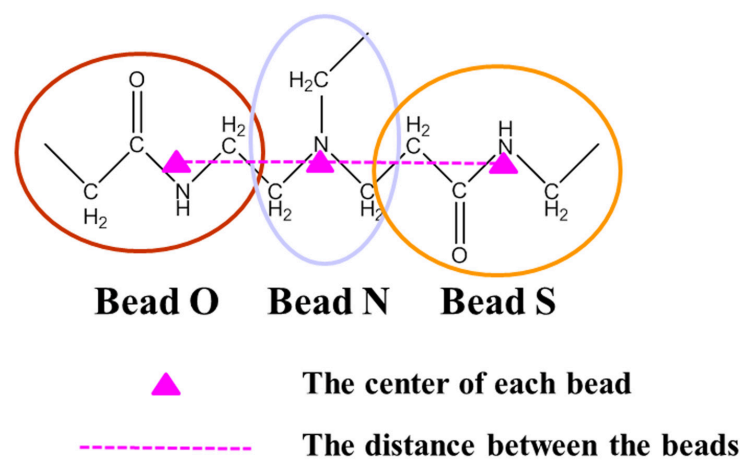

(b)

\subsection{The Physical Properties of Complex Systems of Dendrimers with Guest Molecules in Solution}

The binding chemistry between dendrimers and guest molecules has been studied using coarsegrained simulations to understand the behaviors of complex systems beyond all-atom simulations. As guest molecules, polyelectrolyte polymers (nucleic acids) interacting with dendrimers have been studied using coarse-grained simulations [78,79]. Even though all-atom simulations have been carried out to 
understand the detailed binding mechanisms between nucleic acids and dendrimers at full atomistic resolution $[38,69,72,76]$, there is a high computational cost for all-atom simulations to properly describe these complex systems in solution. Lyulin et al. systematically studied the electrostatic interactions, which varied by the multivalency of the counterions and the linear chain in the explicit solvent condition [78]. The freely jointed bead-spring model was used to model a system containing a G4 dendrimer with 48 positive charges on its surface and a linear polyelectrolyte chain with 10 negatively charged beads. Using this model, the simulations predicted that the structure of the dendrimer shrinks due to the strength of the electrostatic interactions whereas the structure of the polyelectrolyte chain was unchanged. In the complex, the electrostatic interaction between the chain and its counterions was screened by the dendrimer-chain complex, and the dehydration of the chain was occurred by the electrostatic interaction. Therefore they effectively explained the effect of electrostatic interactions by the multivalency of the counterions and the polyelectrolytes in drug and gene delivery. However, there is a discrepancy of the binding position of the polyelectrolyte to the dendrimer compared to the all-atom simulations [69]. In the coarse-grained simulations, the chain beads were located to the center of the dendrimer, but in the all-atom simulations, a ssDNA was located far away from the center of the dendrimer. The main reasons of the discrepancy of the binding position is related to the steric hinderance of the dendrimer and the chain, which can be provided by the introduction of angle and torsional potentials. Tian et al. investigated the effect of the chain rigidity using the freely jointed bead-spring model [79]. In this study, the stiffness of the polyelectrolyte was varied by the strength of bond angle constants. The increase of the linear chain rigidity provided the interesting conformational transformations from coil to $\mathrm{U}$ or $\mathrm{V}$ then to rod shape due to the increase of the bending energy occurred by the chain stiffness. Also they found that the size and the shape of the dendrimer are changed by the stiffness of the charged linear chain and the Bjerrum length, which represents the strength of electrostatic interactions in the system.

To see the interaction of dendrimers, Tian et al. investigated the energy barriers depending on solvent $\mathrm{pH}$, counterions, and modification of terminal groups of dendrimers using the MARTINI coarse-grained model, which is a generalized coarse-grained force field for proteins and cell membranes [92]. For the solvent $\mathrm{pH}$ effect, they found that the decrease of the solvent $\mathrm{pH}$ leads the repulsive interactions between dendrimers stronger due to the electrostatic interactions. In the higher valency of the salt ions, the release of the free energy between dendrimers is getting higher, so the dendrimers are easily aggregated and make more stable clusters. For the modification of the terminal groups of the dendrimers, the aggregation of the dendrimers was not changed even though the stability of the clusters was lower in the charged functional groups.

Our group has studied the interactions between G5 PAMAM dendrimers and phenanthrene molecules using a new coarse-grained modeling scheme that combines a systematic, solvent-free multiscale coarse-graining algorithm for a complex macromolecule with an existing coarse-grained solvent model [49]. The solvent-free coarse-graining approach [84] does not work well for flexible macromolecules in solution because of the significant configurational entropy loss from the absence of explicit solvent molecules. To overcome this problem, the configurational entropy was restored by reintroducing coarse-grained solvent molecules to the system. The new coarse-grained modeling approach predicted the experimentally measured binding capacity and reproduced the distribution 
of phenanthrene molecules obtained with all-atom molecular dynamics simulations (Figure 6). The advantages of this coarse-grained methodology is to obtain the coarse-grained potentials derived from the all-atom simulations for the flexible macromolecules in solution such as drug delivery systems, so the coarse-grained potentials provide better descriptions of the binding mechanism at a specific system even though this method could not provide generic coarse-grained potentials.

Figure 6. Two-dimensional probability distribution function, $\mathrm{P}(\mathrm{r})$, of Phe molecules from the core of each dendrimer. The white circles indicate the branch points of the dendrimers. The numbers in the circles represent the generation of the dendrimer, and $\mathrm{Q}$ is the abbreviation for $Q_{d}$. The color bars express the intensity of $P(r)$. The unit of $X$ and $\mathrm{Y}$ axis is nm. (a) Atomistic simulation; (b) solvent free coarse-grained; (c) explicit solvent coarse-grained (27 dendrimers); (d) explicit solvent coarse-grained (216 dendrimers) Reprinted with permission from [49]. Copyright 2011 by the American Physical Society.

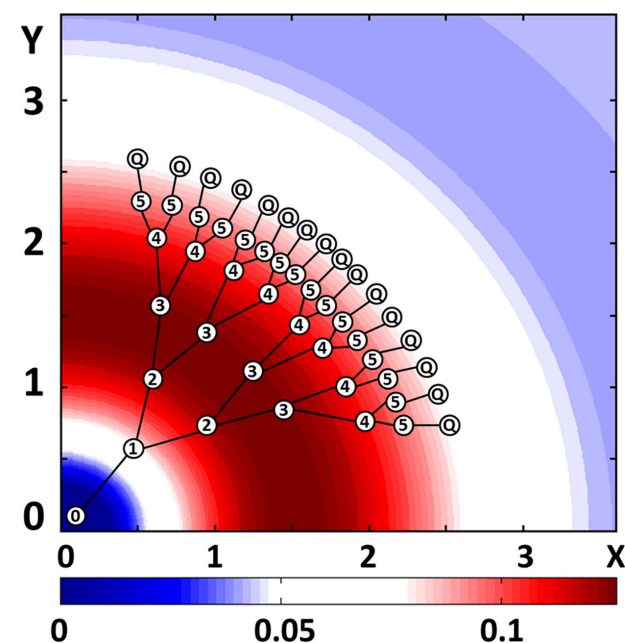

(a)

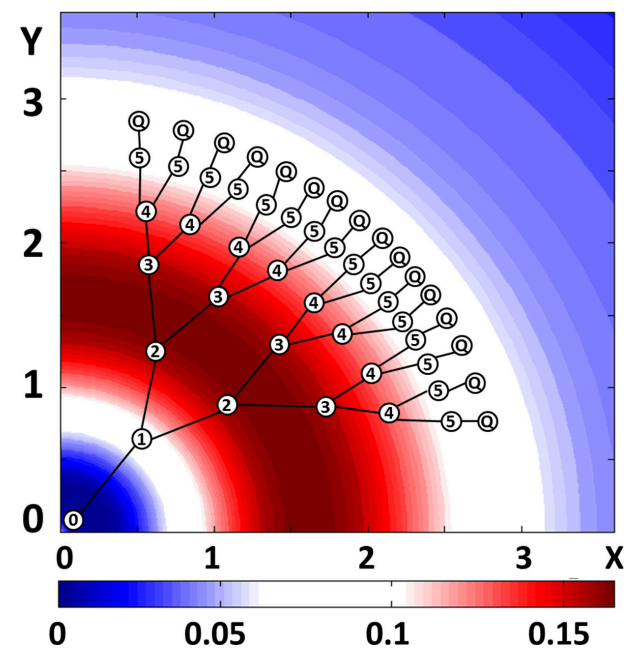

(c)

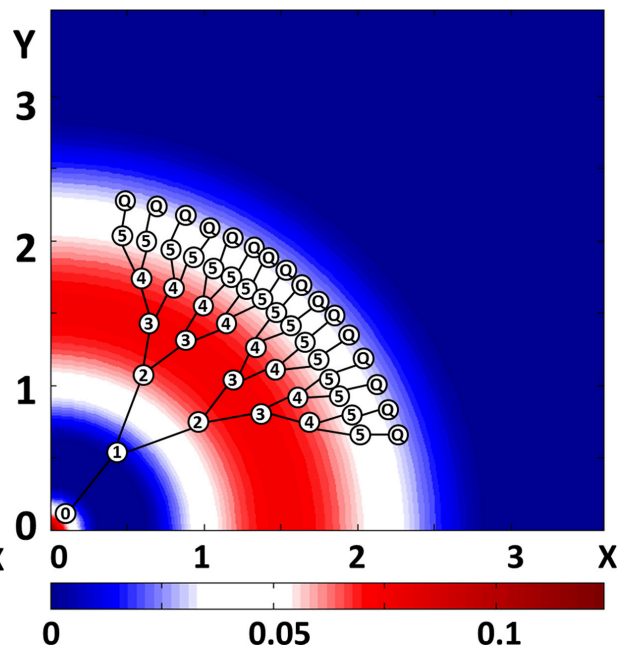

(b)

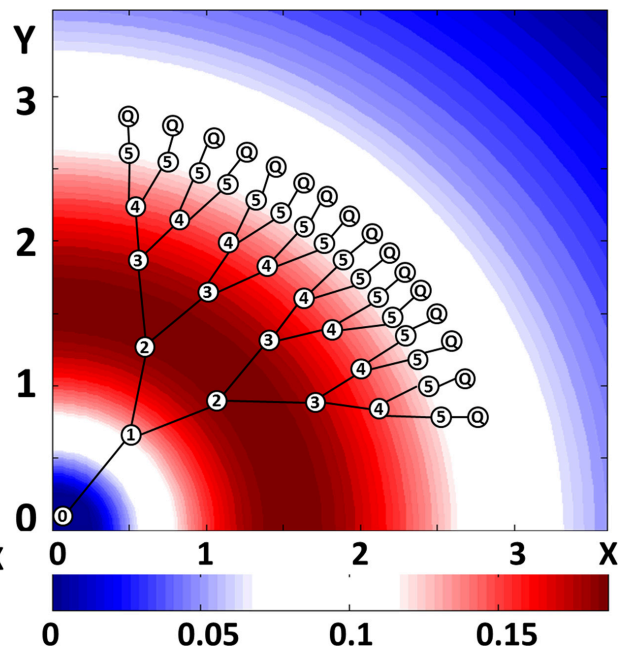

(d)

Coarse-grained simulations provide information about the interaction between dendrimers and guest molecules on a longer characteristic time scale than is possible with all-atom simulations. To ensure accurate and reliable results, it is best when coarse-grained potentials are derived or informed by 
experiments or by higher resolution computational methods such as all-atom simulations or ab-initio quantum mechanics. When coarse-grained potentials are carefully developed, binding information such as the optimal loading of guest molecules or the binding thermodynamics can be obtained. However, dynamic information, such as diffusion, or atomistically detailed information, such as hydrogen bonding, are not readily provided with coarse-grained simulations.

\section{Statistical Field Theories}

Statistical field theories, such as self-consistent field theory (SCFT) or nonlocal density functional theory where the degrees of freedom are fluctuating fields, are an alternative to particle-based methods such as all-atom or coarse-grained molecular dynamics [93]. In particular, SCFT has been widely used for the prediction of equilibrium mesophases in polymeric systems [94-98]. Field-based simulation approaches readily provide the decomposition of free energy into entropic and enthalpic contributions. This is advantageous for modeling the binding mechanism between dendrimers and guest molecules when the balance between entropic and enthalpic terms is desired [96]. However, no kinetic data regarding binding can be obtained from statistical field theories.

Boris and Rubinstein proposed the dense-core model for the equilibrium structure of the starburst dendrimers using a modified SCFT approach [94]. Since that time, the mean field theory has been performed to understand the relationship between enthalpic and entropic driving forces and their influence on the free energy of dendrimer systems. Giupponi et al. studied the conformational change of polyelectrolyte dendrimers as a function of dendrimer generation using molecular dynamics simulations and mean field theory [95]. In this study, the explicit free ions and the implicit solvent models were applied to more accurately describe the electrostatic interaction of the dendrimer rather than the Debye-Hückel approximation, since there are important nonlinear phenomena present in the counterion distribution. Using this model, they found that the variation of the dendrimer structure was weaker with increasing concentration of salt ions than the structure prediction given by the Debye-Hückel approximation. Giupponi et al. explained that the weaker dependence of the dendrimer structure was due to the osmotic pressure of the trapped counterions because the electrostatic interactions were strongly screened by the local charge neutrality. They performed mean field theory calculations to determine the osmotic pressure of the dendrimer based on the free energy calculation. From these calculations, the osmotic brush regime for the dendrimer, in which the electrostatic interactions are strongly screened, dominated to cause swelling of the dendrimer structure. Ting et al. studied hole formation and rupture of a membrane caused by the insertion of a fully charged dendrimer "nanoparticle" using SCFT to rationally design vectors for gene delivery systems [97]. They simplified the system using the discrete Gaussian chain model, considering electrostatic interactions with constraints of one reaction coordinate and a fixed position for the charged dendrimer. Using this simplified model, they considered two different cases for membrane rupture, a tensionless membrane and a membrane under tension, with the latter case being motivated by the proton sponge hypothesis for cell membranes. In the tensionless membrane, the membrane deformed and partially wrapped the dendrimer (Figure 7); no stable pore was formed in the membrane. For the membrane under tension, pore formation of the membrane was stable if the tension exceeded a critical value. They also found that higher generations of the dendrimer provided more stable pore structure in the membrane; this result is consistent with that using coarse-grained simulation 
based on the MARTINI model [99]. Recently, Ting et al. proposed more sophisticated model using SCFT combined with the string method [100] to calculate the minimum energy path to membrane pore formation and rupture [98]. The string method automatically determines the reaction coordinate of the minimum energy path and thus, provides a means by which one may consider the actual nucleation events for the pore formation induced by the dendrimer.

Figure 7. Volume fraction of head monomers, $\phi_{H}$, in cylindrical coordinates $(z v s . r)$ for a tensionless membrane and a fully protonated G5 dendrimer with charge density of $1.55 \mathrm{~nm}^{-3}$ placed at vertical position $z_{F}$. Reprinted from [97], Copyright (2011), with permission from Elsevier.

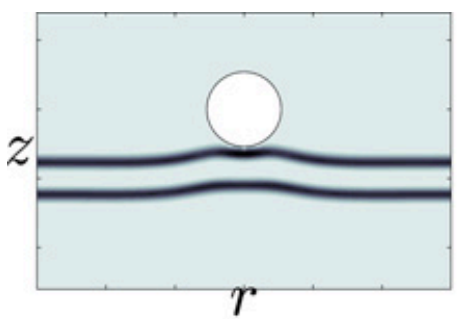

a $z_{\mathrm{F}}=5.0 \mathrm{~nm}$

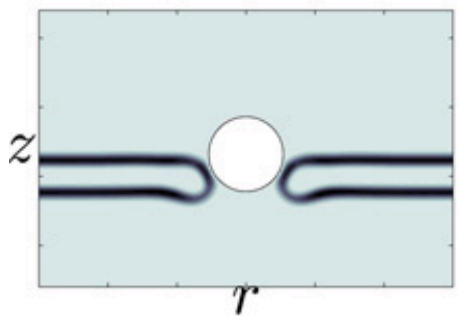

d $z_{\mathrm{F}}=1.6 \mathrm{~nm}$

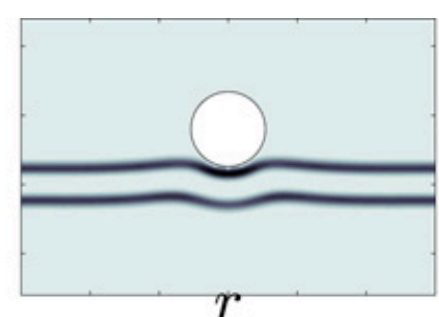

b $z_{\mathrm{F}}=4.0 \mathrm{~nm}$

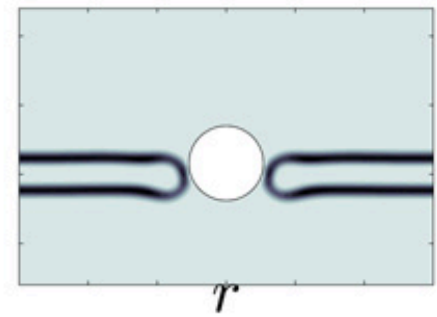

e $z_{\mathrm{F}}=0.8 \mathrm{~nm}$

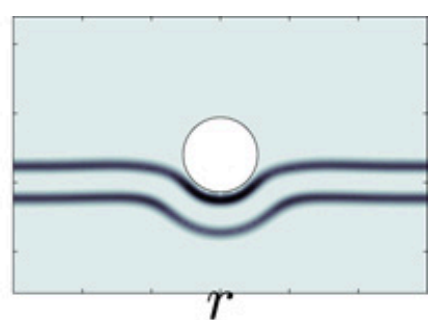

C $z_{\mathrm{F}}=2.0 \mathrm{~nm}$

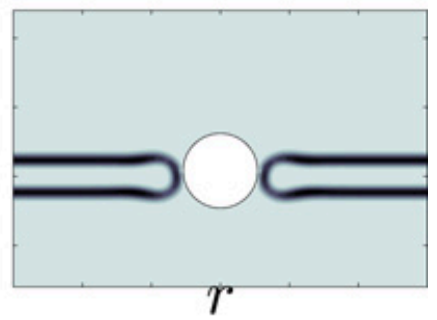

f $z_{\mathrm{F}}=0.2 \mathrm{~nm}$

\section{Conclusions}

The modeling studies discussed here have provided substantial insight into the fundamental mechanisms of dendrimer-guest interaction in solution. Notably, they have shown that solution conditions, including solvent $\mathrm{pH}$ and counterion distribution, play a key role in binding and must be carefully incorporated in the model, whether the simulation is being run at the all-atom, coarse-grained, or mesoscale. In general, when reducing the degrees of freedom to construct multiscale models, great care must be taken to validate the longer length and time scale approaches against experimental data and all-atom simulations or first principles quantum mechanical calculations. Based on the examples discussed here, the outlook is promising for future applications of truly multiscale simulations, that is calculations where one may readily reduce or increase (by reverse-mapping) the degrees of freedom on demand, that address the relevant details of dendrimer-guest binding in solution.

\section{Acknowledgements}

Support from the National Science Foundation (OCI-0749156) is gratefully acknowledged. 


\section{References}

1. Evans, H.M.; Ahmad, A.; Ewert, K.; Pfohl, T.; Martin-Herranz, A.; Bruinsma, R.F.; Safinya, C.R. Structural polymorphism of DNA-dendrimer complexes. Phys. Rev. Lett. 2003, 91, 075501:1-075501:4.

2. Boas, U.; Christensen, J.B.; Heegaard, P.M.H. Dendrimers in Medicine and Biotechnology: New Molecular Tools; Royal Society of Chemistry: Cambridge, UK, 2006.

3. Licata, N.A.; Tkachenko, A.V. Kinetic limitations of cooperativity-based drug delivery systems. Phys. Rev. Lett. 2008, 100, doi:10.1103/PhysRevLett.100.158102.

4. Menjoge, A.R.; Kannan, R.M.; Tomalia, D.A. Dendrimer-based drug and imaging conjugates: Design considerations for nanomedical applications. Drug Discov. Today 2010, 15, 171-185.

5. Navath, R.S.; Menjoge, A.R.; Wang, B.; Romero, R.; Kannan, S.; Kannan, R.M. Amino acid-functionalized dendrimers with heterobifunctional chemoselective peripheral groups for drug delivery applications. Biomacromolecules 2010, 11, 1544-1563.

6. Turrin, C.; Caminade, A. Dendrimers for imaging. In Dendrimers: Towards Catalytic, Material and Biomedical Uses; Caminade, A., Turrin, C., Laurent, R., Ouali, A., Delavaux-Nicot, B., Eds.; John Wiley \& Sons: Hoboken, NJ, USA, 2011; pp. 393-412.

7. Olson, E.S.; Jiang, T.; Aguilera, T.A.; Nguyen, Q.T.; Ellies, L.G.; Scadeng, M.; Tsien, R.Y. Activatable cell penetrating peptides linked to nanoparticles as dual probes for in vivo fluorescence and $\mathrm{mr}$ imaging of proteases. Proc. Natl. Acad. Sci. USA 2010, 107, 4311-4316.

8. Lang, H.; May, R.A.; Iversen, B.L.; Chandler, B.D. Dendrimer-encapsulated nanoparticle precursors to supported platinum catalysts. J. Am. Chem. Soc. 2003, 125, 14832-14836.

9. Yancey, D.F.; Carino, E.V.; Crooks, R.M. Electrochemical synthesis and electrocatalytic properties of Au@Pt dendrimer-encapsulated nanoparticles. J. Am. Chem. Soc. 2010, 132, 10988-10989.

10. Albiter, M.A.; Morales, R.; Zaera, F. Dendrimer-based synthesis of Pt catalysts for hydrocarbon conversion. Appl. Catal. A Gen. 2011, 391, 386-393.

11. Diallo, M.S.; Christie, S.; Swaminathan, P.; Balogh, L.; Shi, X.; Um, W.; Papelis, C.; Goddard, W.A., III.; Johnson, J.H. Dendritic chelating agents. 1. $\mathrm{Cu}(\mathrm{II})$ binding to ethylene diamine core poly(amidoamine) dendrimers in aqueous solutions. Langmuir 2004, 20, 2640-2651.

12. Diallo, M.S.; Christie, S.; Swaminathan, P.; Johnson, J.H.; Goddard, W.A., III. Dendrimer enhanced ultrafiltration. 1. Recovery of $\mathrm{Cu}$ (II) from aqueous solutions using pamam dendrimers with ethylene diamine core and terminal NH2 groups. Environ. Sci. Technol. 2005, 39, 1366-1377.

13. Diallo, M.S.; Falconer, K.; Johnson, J.H. Dendritic anion hosts: Perchlorate uptake by G5-NH2 poly(propyleneimine) dendrimer in water and model electrolyte solutions. Environ. Sci. Technol. 2007, 41, 6521-6527.

14. Diallo, M.S.; Arasho, W.; Johnson, J.H.; Goddard, W.A., III. Dendritic chelating agents. 2. $\mathrm{U}(\mathrm{VI})$ binding to poly(amidoamine) and poly(propyleneimine) dendrimers in aqueous solutions. Environ. Sci. Technol. 2008, 42, 1572-1579. 
15. Tomalia, D.A. Dendrons/dendrimers: Quantized, nano-element like building blocks for soft-soft and soft-hard nano-compound synthesis. Soft Matter 2010, 6, 456-474.

16. Newkome, G.R.; Moorefield, C.N.; Vogtle, F. Dendritic Molecules: Concepts, Syntheses, Perspectives; John Wiley \& Sons: Hoboken, NJ, USA, 1996.

17. Ke, W.; Shao, K.; Huang, R.; Han, L.; Liu, Y.; Li, J.; Kuang, Y.; Ye, L.; Lou, J.; Jiang, C. Gene delivery targeted to the brain using an angiopep-conjugated polyethyleneglycol-modified polyamidoamine dendrimer. Biomaterials 2009, 30, 6976-6985.

18. Peng, S.F.; Su, C.J.; Wei, M.C.; Chen, C.Y.; Liao, Z.X.; Lee, P.W.; Chen, H.L.; Sung, H.W. Effects of the nanostructure of dendrimer/DNA complexes on their endocytosis and gene expression. Biomaterials 2010, 31, 5660-5670.

19. Shcharbin, D.; Dzmitruk, V.; Shakhbazau, A.; Goncharova, N.; Seviaryn, I.; Kosmacheva, S.; Potapnev, M.; Pedziwiatr-Werbicka, E.; Bryszewska, M.; Talabaev, M.; Chernov, A.; Kulchitsky, V.; Caminade, A.M.; Majoral, J.P. Fourth generation phosphorus-containing dendrimers: Prospective drug and gene delivery carrier. Pharmaceutics 2011, 3, 458-473.

20. Nguyen, Q.T.; Olson, E.S.; Aguilera, T.A.; Jiang, T.; Scadeng, M.; Ellies, L.G.; Tsien, R.Y. Surgery with molecular fluorescence imaging using activatable cell-penetrating peptides decreases residual cancer and improves survival. Proc. Natl. Acad. Sci. USA 2010, 107, 4317-4322.

21. Han, L.; Li, J.; Huang, S.; Huang, R.; Liu, S.; Hu, X.; Yi, P.; Shan, D.; Wang, X.; Lei, H.; Jiang, C. Peptide-conjugated polyamidoamine dendrimer as a nanoscale tumor-targeted T1 magnetic resonance imaging contrast agent. Biomaterials 2011, 32, 2989-2998.

22. Jasmine, M.J.; Prasad, E. Fractal growth of PAMAM dendrimer aggregates and its impact on the intrinsic emission properties. J. Phys. Chem. B 2010, 114, 7735-7742.

23. Arkas, M.; Tsiourvas, D.; Paleos, C.M. Functional dendrimeric "nanosponges" for the removal of polycyclic aromatic hydrocarbons from water. Chem. Mater. 2003, 15, 2844-2847.

24. Lard, M.; Kim, S.H.; Lin, S.; Bhattacharya, P.; Ke, P.C.; Lamm, M.H. Fluorescence resonance energy transfer between phenanthrene and PAMAM dendrimers. Phys. Chem. Chem. Phys. 2010, 12, 9285-9291.

25. Astruc, D.; Boisselier, E.; Ornelas, C. Dendrimers designed for functions: From physical, photophysical, and supramolecular properties to applications in sensing, catalysis, molecular electronics, photonics, and nanomedicine. Chem. Rev. 2010, 110, 1857-1959.

26. Kelly, C.V.; Liroff, M.G.; Triplett, L.D.; Leroueil, P.R.; Mullen, D.G.; Wallace, J.M.; Meshinchi, S.; Baker, J.R.; Orr, B.G.; Holl, M.M.B. Stoichiometry and structure of poly(amidoamine) dendrimer-lipid complexes. ACS Nano 2009, 3, 1886-1896.

27. Yang, W.; Li, Y.; Cheng, Y.; Wu, Q.; Wen, L.; Xu, T. Evaluation of phenylbutazone and poly(amidoamine) dendrimers interactions by a combination of solubility, 2D-NOESY NMR, and isothermal titration calorimetry studies. J. Pharm. Sci. 2009, 98, 1075-1085.

28. Jensen, L.B.; Mortensen, K.; Pavan, G.M.; Kasimova, M.R.; Jensen, D.K.; Gadzhyeva, V.; Nielsen, H.M.; Foged, C. Molecular characterization of the interaction between siRNA and PAMAM G7 dendrimers by SAXS, ITC, and Molecular dynamics simulations. Biomacromolecules 2010, 11, 3571-3577. 
29. Bhattacharya, P.; Kim, S.H.; Chen, P.; Chen, R.; Spuches, A.M.; Brown, J.M.; Lamm, M.H.; Ke, P.C. Complexation of PAMAM dendrimer and fullerenol for drug delivery and environmental remediation. unpublished

30. Shi, X.; Bi, X.; Ganser, T.R.; Hong, S.; Myc, L.A.; Desai, A.; Holl, M.M.B.; Baker, J.R. HPLC analysis of functionalized poly(amidoamine) dendrimers and the interaction between a folate-dendrimer conjugate and folate binding protein. Analyst 2006, 131, 842-848.

31. Orberg, M.; Schillen, K.; Nylander, T. Dynamic light scattering and fluorescence study of the interaction between double-stranded DNA and poly(amido amine) dendrimers. Biomacromolecules 2007, 8, 1557-1563.

32. Froehlich, E.; Mandeville, J.S.; Jennings, C.J.; Sedaghat-Herati, R.; Tajmir-Riahi, H.A. Dendrimers bind human serum albumin. J. Phys. Chem. B 2009, 113, 6986-6993.

33. Mandeville, J.S.; Tajmir-Riahi, H.A. Complexes of dendrimers with bovine serum albumin. Biomacromolecules 2010, 11, 465-472.

34. Hu, J.; Cheng, Y.; Ma, Y.; Wu, Q.; Xu, T. Host-guest chemistry and physicochemical properties of the dendrimer-mycophenolic acid complex. J. Phys. Chem. B 2009, 113, 64-74.

35. Hu, J.; Cheng, Y.; Wu, Q.; Zhao, L.; Xu, T. Host-guest chemistry of dendrimer-drug complexes. 2. Effects of molecular properties of guests and surface functionalities of dendrimers. J. Phys. Chem. B 2009, 113, 10650-10659.

36. Montanez, M.I.; Najera, F.; Perez-Inestrosa, E. NMR studies and molecular dynamic simulation of synthetic dendritic antigens. Polymers 2011, 3, 1533-1553.

37. Lee, H.; Larson, R. Multiscale modeling of dendrimers and their interactions with bilayers and polyelectrolytes. Molecules 2009, 14, 423-438.

38. Nandy, B.; Maiti, P.K. DNA compaction by a dendrimer. J. Phys. Chem. B 2011, 115, 217-230.

39. Jayamurugan, G.; Vasu, K.S.; Rajesh, Y.B.R.D.; Kumar, S.; Vasumathi, V.; Maiti, P.K.; Sood, A.K.; Jayaraman, N. Interaction of single-walled carbon nanotubes with poly(propyl ether imine) dendrimers. J. Chem. Phys. 2011, 134, 104507:1-104507:6.

40. Voth, G.A. Coarse-Graining of Condensed Phase and Biomolecular Systems, 1st ed.; CRC Press: Boca Raton, FL, USA, 2008.

41. Lin, C.; Wu, K.; Sa, R.; Mang, C.; Liu, P.; Zhuang, B. Density functional theory studies on the potential energy surface and hyperpolarizability of polyamidoamide dendrimer. Chem. Phys. Lett. 2002, 363, 343-348.

42. Tarazona-Vasquez, F.; Balbuena, P.B. Complexation of the lowest generation poly(amidoamine)NH2 dendrimers with metal ions, metal atoms, and $\mathrm{Cu}$ (II) hydrates: An ab initio study. J. Phys. Chem. B 2004, 108, 15992-16001.

43. Wan, H.; Li, S.; Konovalova, T.A.; Shuler, S.F.; Dixon, D.A.; Street, S.C. Experimental and theoretical studies of the photoreduction of copper(II)-dendrimer complexes. J. Phys. Chem. C 2008, 112, 1335-1344.

44. Kitaura, K.; Ikeo, E.; Asada, T.; Nakano, T.; Uebayasi, M. Fragment molecular orbital method: An approximate computational method for large molecules. Chem. Phys. Lett. 1999, 313, 701-706. 
45. Fedorov, D.G.; Kitaura, K. Extending the power of quantum chemistry to large systems with the fragment molecular orbital method. J. Phys. Chem. A 2007, 111, 6904-6914.

46. Fedorov, D.G.; Kitaura, K. Pair interaction energy decomposition analysis. J. Comput. Chem. 2007, 28, 222-237.

47. Fedorov, D.G.; Kitaura, K.; Li, H.; Jensen, J.H.; Gordon, M.S. The polarizable continuum model (PCM) interfaced with the fragment molecular orbital method (FMO). J. Comput. Chem. 2006, 27, 976-985.

48. Maiti, P.K.; Messina, R. Counterion distribution and $\zeta$-potential in PAMAM dendrimer. Macromolecules 2008, 41, 5002-5006.

49. Kim, S.H.; Lamm, M.H. Reintroducing explicit solvent to a solvent-free coarse-grained model. Phys. Rev. E 2011, 84, 025701:1-025701:4.

50. Tanis, I.; Karatasos, K. Association of a weakly acidic anti-inflammatory drug (ibuprofen) with a poly(amidoamine) dendrimer as studied by molecular dynamics simulations. J. Phys. Chem. B 2009, 113, 10984-10993.

51. Shi, X.; Lee, I.; Chen, X.; Shen, M.; Xiao, S.; Zhu, M.; Baker, J.R.; Wang, S.H. Influence of dendrimer surface charge on the bioactivity of 2-methoxyestradiol complexed with dendrimers. Soft Matter 2010, 6, 2539-2545.

52. Maiti, P.K.; Cagin, T.; Lin, S.; Goddard, W.A., III. Effect of solvent and pH on the structure of PAMAM dendrimers. Macromolecules 2005, 38, 979-991.

53. Han, M.; Chen, P.; Yang, X. Molecular dynamics simulation of PAMAM dendrimer in aqueous solution. Polymer 2005, 46, 3481-3488.

54. Lee, H.; Baker, J.R.; Larson, R.G. Molecular dynamics studies of the size, shape, and internal structure of $0 \%$ and $90 \%$ acetylated fifth-generation polyamidoamine dendrimers in water and methanol. J. Phys. Chem. B 2006, 110, 4014-4019.

55. Giri, J.; Diallo, M.S.; Goddard, W.A., III.; Dalleska, N.F.; Fang, X.; Tang, Y. Partitioning of poly(amidoamine) dendrimers between n-octanol and water. Environ. Sci. Technol. 2009, 43, 5123-5129.

56. Weiner, P.K.; Kollman, P.A. AMBER: Assisted model building with energy refinement. A general program for modeling molecules and their interactions. J. Comput. Chem. 1981, 2, 287-303.

57. Wang, J.; Wolf, R.M.; Caldwell, J.W.; Kollman, P.A.; Case, D.A. Development and testing of a general amber force field. J. Comput. Chem. 2004, 25, 1157-1174.

58. Brooks, B.R.; Bruccoleri, R.E.; Olafson, B.D.; States, D.J.; Swaminathan, S.; Karplus, M. CHARMM: A program for macromolecular energy, minimization, and dynamics calculations. J. Comput. Chem. 1983, 4, 187-217.

59. Pissurlenkar, R.R.; Shaikh, M.S.; Iyer, R.P.; Coutinho, E.C. Molecular mechanics force fields and their applications in drug design. Anti-Infect. Agents Med. Chem. 2009, 8, 128-150.

60. Dauber-Osguthorpe, P.; Roberts, V.A.; Osguthorpe, D.J.; Wolff, J.; Genest, M.; Hagler, A.T. Structure and energetics of ligand binding to proteins: Escherichia coli dihydrofolate reductase-trimethoprim, a drug-receptor system. Proteins Struct. Funct. Bioinform. 1988, 4, $31-47$. 
61. Mayo, S.L.; Olafson, B.D.; Goddard, W.A., III. DREIDING: A generic force field for molecular simulations. J. Phys. Chem. 1990, 94, 8897-8909.

62. Maiti, P.K.; Goddard, W.A., III. Solvent quality changes the structure of G8 pamam dendrimer, a disagreement with some experimental interpretations. J. Phys. Chem. B 2006, 110, 25628-25632.

63. Liu, Y.; Bryantsev, V.S.; Diallo, M.S.; Goddard, W.A., III. Pamam dendrimers undergo pH responsive conformational changes without swelling. J. Am. Chem. Soc. 2009, 131, 2798-2799.

64. Welch, P.; Muthukumar, M. Dendrimer-polyelectrolyte complexation: A model guest-host system. Macromolecules 2000, 33, 6159-6167.

65. Lee, I.; Athey, B.D.; Wetzel, A.W.; Meixner, W.; Baker, J.R. Structural molecular dynamics studies on polyamidoamine dendrimers for a therapeutic application: Effects of $\mathrm{pH}$ and generation. Macromolecules 2002, 35, 4510-4520.

66. Lyulin, S.V.; Evers, L.J.; van der Schoot, Paul; Darinskii, A.A.; Lyulin, A.V.; Michels, M.A.J. Effect of solvent quality and electrostatic interactions on size and structure of dendrimers. Brownian dynamics simulation and mean-field theory. Macromlecules 2004, 37, 3049-3063.

67. Maiti, P.K.; Bagchi, B. Diffusion of flexible, charged, nanoscopic molecules in solution: Size and pH dependence for PAMAM dendrimer. J. Chem. Phys. 2009, 131, 214901.

68. Pranami, G.; Lamm, M.H.; Vigil, R.D. Molecular dynamics simulation of fractal aggregate diffusion. Phys. Rev. E 2010, 82, 051402:1-051402:10.

69. Maiti, P.K.; Bagchi, B. Structure and dynamics of DNA-dendrimer complexation: Role of counterions, water, base pair sequence. Nano Lett. 2006, 6, 2478-2485.

70. Mills, M.; Orr, B.; Holl, M.M.B.; Andricioaei, I. Microscopic basis for the mesoscopic extensibility of dendrimer-compacted DNA. Biophys. J 2010, 98, 834-842.

71. Ouyang, D.; Zhang, H.; Parekh, H.S.; Smith, S.C. Structure and dynamics of multiple cationic vectors-siRNA complexation by all-atomic molecular dynamics simulations. J. Phys. Chem. B 2010, 114, 9231-9237.

72. Vasumathi, V.; Maiti, P.K. Complexation of siRNA with dendrimer: A molecular modeling approach. Macromolecules 2010, 43, 8264-8274.

73. Ouyang, D.; Zhang, H.; Parekh, H.S.; Smith, S.C. The effect of pH on PAMAM dendrimer-siRNA complexation - Endosomal considerations as determined by molecular dynamics simulation. Biophys. Chem. 2011, 158, 126-133.

74. Jorgensen, W.L.; Chandrasekhar, J.; Madura, J.D.; Impey, R.W.; Klein, M.L. Comparision of simple potential functions for simulating liquid water. J. Chem. Phys. 1983, 79, 926-935.

75. Lyulin, S.V.; Darinskii, A.A.; Lyulin, A.V. Computer simulation of complexes of dendrimers with linear polyelectrolytes. Macromlecules 2005, 38, 3990-3998.

76. Pavan, G.M.; Albertazzi, L.; Danani, A. Ability to adapt: Different generations of PAMAM dendrimers show different behaviors in binding siRNA. J. Phys. Chem. B 2010, 114, 2667-2675.

77. Suek, N.W.; Lamm, M.H. Effect of terminal group modification on the solution properties of dendrimers: A molecular dynamics simulation study. Macromolecules 2006, 39, 4247-4255.

78. Lyulin, S.V.; Vattulainen, I.; Gurtovenko, A.A. Complexes comprised of charged dendrimers, linear polyelectrolytes, and counterions: Insight through coarse-grained molecular dynamics simulations. Macromolecules 2008, 41, 4961-4968. 
79. Tian, W.; Ma, Y. Complexation of a linear polyelectrolyte with a charged dendrimer: Polyelectrolyte stiffness effects. Macromolecules 2010, 43, 1575-1582.

80. Tian, W.; Ma, Y. Effects of valences of salt ions at various concentrations on charged dendrimers. Soft Matter 2010, 6, 1308-1316.

81. Marrink, S.J.; Risselada, H.J.; Yefimov, S.; Tieleman, D.P.; de Vries, A.H. The martini force field: Coarse grained model for biomolecular simulations. J. Phys. Chem. B 2007, 111, 7812-7824.

82. Monticelli, L.; Kandasamy, S.K.; Periole, X.; Larson, R.G.; Tieleman, D.P.; Marrink, S. The MARTINI coarse-grained force field: Extension to proteins. J. Chem. Theory Comput. 2008, 4, 819-834.

83. Izvekov, S.; Voth, G.A. Multiscale coarse graining of liquid-state systems. J. Chem. Phys. 2005, 123, 134105-134113.

84. Izvekov, S.; Voth, G.A. Solvent-free lipid bilayer model using multiscale coarse-graining. J. Phys. Chem. B 2009, 113, 4443-4455.

85. Lu, L.; Izvekov, S.; Das, A.; Andersen, H.C.; Voth, G.A. Efficient, regularized, and scalable algorithms for multiscale coarse-graining. J. Chem. Theory Comput. 2010, 6, 954-965.

86. Larin, S.; Lyulin, S.; Lyulin, A.; Darinskii, A. Charge inversion of dendrimers in complexes with linear polyelectrolytes in the solutions with low pH. Polym. Sci. Ser. A 2009, 51, 459-468.

87. Larin, S.V.; Darinskii, A.A.; Lyulin, A.V.; Lyulin, S.V. Linker formation in an overcharged complex of two dendrimers and linear polyelectrolyte. J. Phys. Chem. B 2010, 114, 2910-2919.

88. Yan, L.; Yu, X. Charged dendrimers on lipid bilayer membranes: Insight through dissipative particle dynamics simulations. Macromolecules 2009, 42, 6277-6283.

89. Maiti, P.K.; Li, Y.; Cagin, T.; Goddard, W.A., III. Structure of polyamidoamide dendrimers up to limiting generations: A mesoscale description. J. Chem. Phys. 2009, 130, 144902.

90. Lee, H.; Larson, R.G. Molecular dynamics simulations of PAMAM dendrimer-induced pore formation in DPPC bilayers with a coarse-grained model. J. Phys. Chem. B 2006, 110, 18204-18211.

91. Maiti, P.K.; Cagin, T.; Wang, G.; Goddard, W.A., III. Structure of pamam dendrimers: Generations 1 through 11. Macromolecules 2004, 37, 6236-6254.

92. Tian, W.; Ma, Y. Coarse-grained molecular simulation of interacting dendrimers. Soft Matter 2011, 7, 500-505.

93. Fredrickson, G.H. The Equilibrium Theory of Inhomogeneous Polymers; Oxford University Press: New York, NY, USA, 2006.

94. Boris, D.; Rubinstein, M. A self-consistent mean field model of a starburst dendrimer: Dense core vs. dense shell. Macromolecules 1996, 29, 7251-7260.

95. Giupponi, G.; Buzza, D.M.A.; Adolf, D.B. Are polyelectrolyte dendrimers stimuli responsive? Macromolecules 2007, 40, 5959-5965.

96. Kim, S.H.; Cochran, E.W. Localization of spherical nanoparticles within lamellar AB diblock copolymer melts through self-consistent field theory. Polymer 2011, 52, 2328-2339.

97. Ting, C.L.; Wang, Z. Interactions of a charged nanoparticle with a lipid membrane: Implications for gene delivery. Biophys. J. 2011, 100, 1288-1297. 
98. Ting, C.L.; Appelo, D.; Wang, Z. Minimum energy path to membrane pore formation and rupture. Phys. Rev. Lett. 2011, 106, 168101:1-168101:4.

99. Lee, H.; Larson, R.G. Lipid bilayer curvature and pore formation induced by charged linear polymers and dendrimers: The effect of molecular shape. J. Phys. Chem. B 2008, 112, 12279-12285.

100. E, W.; Ren, W.; Vanden-Eijnden, E. String method for the study of rare events. Phys. Rev. $B$ 2002, 66, 052301.

(c) 2012 by the authors; licensee MDPI, Basel, Switzerland. This article is an open access article distributed under the terms and conditions of the Creative Commons Attribution license (http://creativecommons.org/licenses/by/3.0/). 